\title{
Desde el Movimiento de Vida Independiente hasta la asistencia personal: los derechos de las personas con diversidad funcional ${ }^{1}$
}

\author{
Mercedes López Pérez \\ UNIVERSIDAD DE JAÉN, JAÉN, ESPAÑA \\ perez@ujaen.es \\ Susana Ruiz Seisdedos \\ UNIVERSIDAD DE JAÉN, JAÉN, ESPAÑA \\ suruiz@ujaen.es
}

Resumen: El objetivo de este artículo es realizar un estudio teórico del primer movimiento social protagonizado por las propias personas con diversidad funcional, el denominado Movimiento de Vida Independiente (MVI), así como uno de los recursos que originó, la denominada asistencia personal (AP), un recurso poco conocido en nuestro país. La asistencia personal es un apoyo fundamental para poner en práctica el derecho a una vida independiente de las personas con diversidad funcional.

Este estudio pretende profundizar en el conocimiento de este movimiento, analizando su origen, desarrollo, su filosofía de vida, sus demandas y reivindicaciones, en primer lugar, a nivel mundial y, en segundo lugar, en España, en los últimos años. Entre las demandas de dicho movimiento se encuentra el empoderamiento de las personas con diversidad funcional a través de la asistencia personal por lo que se analizarán sus funciones, tareas, requisitos y beneficiarios.

Debido a que actualmente existe un escaso desarrollo académico de esta temática en nuestro país y que, en lo últimos años, ha habido un creciente interés en esta figura laboral por parte de las propias personas con diversidad funcional, por las organizaciones del sector y por las administraciones públicas, se ha realizado una revisión bibliográfica y documental exhaustiva.

1. Antes de continuar, es necesario hacer una breve aclaración respecto al uso del concepto "persona con diversidad funcional" en este artículo.

Si bien, la expresión utilizada hasta este momento, en la mayoría de las legislaciones vigentes y en el mundo académico es "personas con discapacidad", a lo largo de este trabajo se ha empleado principalmente la de "persona con diversidad funcional", por varios motivos:

- Surge de las propias personas con diversidad funcional como alternativa a los utilizados hasta el momento.

- Por primera vez se utiliza un término en positivo.

- Centra la atención en la diversidad, como valor enriquecedor para la sociedad.

- Hace referencia a una cuestión de derechos humanos que poco tiene que ver con el estado de salud.

- Es el resultado de un cambio de paradigma.

En definitiva y de acuerdo con láñez (2010) “...es un término que se adecua más acertadamente a la realidad actual y denota una mayor neutralidad en relación a los conceptos utilizados hasta el momento” (p. 18-19). 
Palabras clave: Asistencia personal, movimiento de vida independiente, asistente personal, centro de vida independiente, personas con diversidad funcional

Abstract: The objective of this article is to carry out a theoretical study of the first social movement in which people with disability have taken the leading role, the so-called independent living movement (ILM), as well as one of the resources that it originated: personal assistance (PA), not very well-known in our country. Personal assistance proves to be a fundamental support in order to put into practice the right of people with disability to live an independent living.

This study aims to deepen into the knowledge of this movement, by analysing its origin, development, philosophy of life and its demands and claims, firstly, worldwide, and, secondly, in Spain, in recent years. Among the demands from this movement there is the empowerment of people with disability by means of the resource of personal assistance, so their functions, tasks, requirements and beneficiaries will be analysed.

Due to the fact that there is currently little academic development of this subject in our country, although there has been a growing interest in this labour figure by people with disability by organizations within this area and by public administrations, it has been done an exhaustive bibliographic and documentary review.

Keywords: Personal assistance; independent living movement; personal assistant; independent living center; people with disability

\section{Introducción}

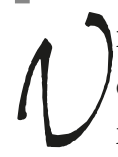

ivimos en una sociedad en la que todos nos necesitamos, nadie es autosuficiente pero esta situación que es obvia para la mayoría de las personas, es un motivo de exclusión para una parte de la población. Más concretamente para todas aquellas personas que por su diversidad funcional no pueden realizar algunas de las actividades de su vida diaria. Esto hace que la independencia o el derecho a vivir de forma independiente se convierta, a veces, en una quimera.

Aún continúa muy extendida una actitud de subestimación hacia estas personas que considera que son incapaces de gestionar sus vidas. En oposición a estas situaciones de marginalidad y exclusión, nace el Movimiento de Vida Independiente (en adelante MVI) (Independent Living Movement, ILM, en su versión original). Este movimiento defiende que la inclusión de las personas con diversidad funcional en la sociedad sólo es posible si son consideradas portadoras de derechos.

En este sentido, los Movimientos Sociales (en adelante MS) deben ser considerados como agentes de influencia y persuasión que vienen a ensanchar el espacio decisorio en distintas políticas públicas. Los MS pretenden interferir -cambiar o mantener o anular- determinadas decisiones política. El MVI ha permitido mirar a las personas con discapacidad desde otra perspectiva, menos paternalista, en la 
que son personas con derechos y que únicamente necesitan un entorno accesible o figuras como la asistencia personal (apoyo humano) para hacer realidad la vida independiente y la participación de estas personas en la sociedad.

\section{Movimiento de Vida Independiente}

Considera Raschke (citado en Dalton, Kuechler y Bürklin, 1992) que los movimientos sociales son actores colectivos que se movilizan para producir un cambio social, que reinventan la democracia, intentando que un número, cada vez mayor, de intereses que no encuentran acomodo en las estructuras o instituciones existentes, principalmente a través de los partidos políticos, hagan oír sus demandas.

Desde este posicionamiento debemos estudiar el Movimiento de Vida Independiente que surge en los Estados Unidos de América a finales de la década de los 60 y primera mitad de los 70 del siglo XX, época de constantes revueltas y reivindicaciones de todo tipo, siendo las propias personas con diversidad funcional las que se agruparon para reivindicar sus derechos y libertades bajo el lema Nothing About Us Without Us (Nada sobre nosotros sin nosotros). Éstas reclaman el derecho a la vida independiente de todas las personas con diversidad funcional, con los apoyos que sean necesarios (Arnau, 2013; García Alonso, 2003; Madrid Pérez, 2013). También se caracteriza por redefinir el fenómeno de la discapacidad, lo que dio lugar a nuevos modelos de servicios y prestaciones (Dejong, 1979).

Ed Roberts es considerado el padre del MVI (Dejong, 1979; Iáñez, 2009). Fue la primera persona con una gran diversidad funcional y con grandes necesidades de apoyo admitido como estudiante en la Universidad de Berkeley (California) en el año 1962. Fueron muchos los obstáculos que tuvo que superar para acceder a los estudios superiores, debido a la idea de subestimación que existía en aquellos años.

En el año 1970, Ed Roberts se asoció con otros estudiantes con diversidad funcional de la citada universidad para crear el Programa de Estudiantes Discapacitados Físicos (Physically Disabled Student's Program), iniciándose así el desarrollo del MVI (Palacios y Romañach, 2006 y Urmeneta, 2007). Con este programa demandaron algunas mejoras en el campus universitario como mayor accesibilidad, transporte adaptado por el campus, talleres para reparar las ayudas técnicas y asistencia personal. Dos años después, este grupo de estudiantes con diversidad funcional fundó el primer Centro de Vida Independiente (en adelante CVI) (Independent Living Center, en su versión original), en la misma universidad. En los años siguientes, se crearon otros centros fuera de la universidad, comenzando así la expansión por todo el territorio estadounidense. En este sentido, se fundaron CVI's en California, Houston, Boston, New York y Chicago. En la actualidad existen más de 400 en los Estados Unidos de América (García Alonso, 2003). 


\subsection{Filosofía del Movimiento de Vida Independiente}

El MVI se asienta en una corriente filosófica internacional que define la diversidad funcional, delimita el problema que pueda significar, busca las soluciones a ese posible problema y señala a los responsables para dar soluciones. Esta filosofía se ha convertido en una nueva corriente teórico-práctica alrededor del fenómeno de la diversidad funcional, donde el entorno socio-político, económico, arquitectónico y de comunicación es el que discapacita. Por lo cual, la reivindicación de los derechos humanos por parte de las personas con diversidad funcional, es la forma de abandonar la situación de discriminación en que viven (Arnau, 2006; García-Alonso, 2003). Irreversiblemente, esta filosofía origina una voluntad de cambio en las políticas y en la legislación que favorece una actitud nueva frente al fenómeno de la diversidad funcional (Maraña y Ratzka, 1999).

Esta filosofía, inspirada en los principios del empowerment, parte de la idea que todas las personas tienen derecho a la independencia, a través del máximo control sobre sus vidas, basado en la capacidad y la oportunidad para tomar decisiones y llevar a cabo las actividades de cada día (Iañez, 2009). Son principios básicos y universales de esta filosofía (García Alonso, 2003; Lobato, 2005):

- Garantizar los derechos humanos y civiles.

- Autodeterminación de las personas para decidir sobre su propio destino.

- Autoayuda o apoyo entre iguales.

- Posibilidad para ejercer poder (Empoderamiento).

- Derecho a asumir riesgos.

- Vivir en la comunidad.

- Responsabilidad sobre la propia vida y acciones.

Para alcanzar estos principios básicos y conseguir las condiciones necesarias para lograr una vida independiente, este movimiento demanda (Centeno, Lobato y Romañach, 2008):

- Un servicio de asistencia personal auto-gestionado, eficaz y asequible a la economía de cada persona con diversidad funcional.

- Ayudas económicas en forma de pago directo.

- La accesibilidad del entorno y ayudas técnicas para lograr una verdadera autodeterminación.

- Una educación inclusiva que posibilite un desarrollo personal y social.

- El derecho a vivir su propia sexualidad y a poder formar una familia. 
Esta filosofía surgió basándose en estas tres ideas: des-institucionalización, desmedicalización y des-profesionalización (Arnau, 2006) ${ }^{2}$.

Desde este nuevo enfoque, las personas con diversidad funcional tienen un papel activo y participativo en la sociedad, en contraposición a la tradicional imagen pasiva o paciente que se ha dado de ellas.

La idea de vida independiente de este movimiento ha tenido una gran influencia en las políticas sobre discapacidad en todo el mundo. De acuerdo con lo aportado por Hasler (2003) este concepto es, a la vez, apolítico y político. Por un lado, es una concepción apolítica porque interesa directamente a los responsables de las políticas sociales cualquiera que sea su ideología. Y por otro, también es un concepto político, ya que exige cambios culturales y en el entorno para apoyar la independencia de las personas con diversidad funcional.

En síntesis, la filosofía de vida independiente se basa en un movimiento de personas que reclaman sus derechos sin intermediarios, en cooperación y ayuda mutua (Gómez Jiménez, 2012) y el servicio de asistencia personal se ha convertido en una pieza clave para conseguirlo.

\subsection{Centros de Vida Independiente}

El primer CVI se crea en el año 1972, en la universidad de Berkeley. Entre sus objetivos se encuentran que las propias personas con diversidad funcional puedan defender sus derechos y tengan el control sobre sus propias vidas (Novoa, 2003). Para el MVI, estos centros son una "[...] herramienta de cooperación mutua y desarrollo comunitario para promover la vida independiente y poner en práctica los principios de esta filosofía a nivel local” (Gómez y Arroyo, 2013: 84).

Aunque todos tienen como nexo de unión su filosofía, existen diferencias entre ellos en cuanto a la organización, los servicios que ofrecen, la financiación, etc. Todo ello va a depender de las características geográficas, históricas, culturales, de las necesidades de la población, de la política y de la legislación en materia de autonomía personal. Sin embargo, todos los CVI's tienen en común que son organizaciones sin ánimo de lucro (asociaciones, cooperativas sociales, fundaciones, etc.), no residenciales, dirigidas y gestionadas por personas con diversidad funcional y orientadas a

2. Des-institucionalización: Las personas con diversidad funcional tienen derecho a vivir en un entorno normalizado con los apoyos que sean necesarios.

Des-medicalización: Es un rechazo al planteamiento médico-rehabilitador, donde las personas con diversidad funcional dejen de ser vistas como enfermas.

Des-profesionalización: Las personas con diversidad funcional son las dueñas de sus propias vidas y ellas, mejor que nadie, saben de la realidad de la diversidad funcional. Pasando los profesionales a un plano de igualdad con respecto a las personas con diversidad funcional. 
facilitar apoyos y servicios que hagan posible el diseño de planes individuales de vida independiente y auto-determinada (Iañez, 2009; Novoa, 2003).

El objetivo fundamental es empoderar a las personas con diversidad funcional, para que sean capaces de organizar los servicios y apoyos necesarios para ejercer el derecho a controlar y a adquirir la responsabilidad sobre sus propias vidas y lograr unos niveles de actividad y participación en la sociedad como el resto de los ciudadanos (Maraña, 2004).

Por lo general, estos centros proporcionan servicios y recursos a todas las personas con cualquier tipo de diversidad funcional que necesiten una ayuda continuada para realizar sus actividades. La gran mayoría son servicios esenciales para facilitar una vida autónoma y la plena inclusión en la comunidad de estas personas. Entre ellos están (Gómez y Arroyo, 2013):

- Información y asesoramiento: Es un servicio de orientación sobre los recursos y ayudas sociales para facilitar la independencia.

- Formación en habilidades para la vida independiente: Su objetivo es enseñar y desarrollar destrezas que promuevan la autodeterminación y la mejora de la calidad de vida.

- Ayuda entre iguales: Es el proceso de orientación, formación y motivación que proporciona una persona con diversidad funcional y con experiencia en vida independiente a otra persona con diversidad funcional que no la tenga.

- Cursos y talleres: Es práctica habitual que los CVI realicen cursos, charlas, talleres, seminarios sobre cuestiones del MVI, por ejemplo, a través de la preparación de documentación para divulgar su filosofía.

- Servicio de asistencia personal auto-gestionado: Puede estar subvencionado por la administración pública o por los propios usuarios del servicio. Va dirigido a la persona con diversidad funcional y con necesidades de apoyo, proporcionándole bienestar, ayuda, comodidad, seguridad e inclusión dentro de la comunidad local y la sociedad, facilitándole apoyo personal en distintas áreas de la vida como en la higiene, la movilidad exterior y el hogar.

- Servicio de formación y orientación laboral: Su misión es facilitar el acceso al empleo de las personas con diversidad funcional y la búsqueda de asistentes personales a través del manteniendo de una bolsa de empleo de personas interesadas en trabajar en esta profesión.

\subsection{Movimiento de Vida Independiente en Europa}

No fue hasta los últimos años de la década de los 70 y los primeros de los 80 que el MVI llegó a Europa. Comenzó introduciéndose por las islas británicas, los países 
nórdicos y de ahí se extendió al resto del continente. Años más tarde, como consecuencia de su expansión y desarrollo por Europa, se aprobaron las primeras normativas específicas sobre asistencia personal y vida independiente en países como Suecia, Reino Unido, Italia y Noruega (Campo, 2005).

Los precursores del MVI europeo fueron personas que previamente trasladaron sus residencias durante un tiempo a los Estados Unidos con objeto de aprender todo lo relacionado con este movimiento y poder implantarlo posteriormente, en sus respectivos países.

Se atribuyen a John Evans en el Reino Unido y a Adolf Radzka en Suecia, los inicios en Europa (Rodríguez-Picavea, 2010). Gracias a ellos, las personas con diversidad funcional en este continente fueron tomando conciencia de sus derechos y se agruparon creando numerosos CVI, cooperativas, plataformas, foros, etc. Consecuentemente, a nivel europeo, se formó la Red Europea de Vida Independiente (European Network of Independent Living, ENIL), el Foro Europeo de la Discapacidad (European Disability Forum), la Coalición Europea para la Vida en Comunidad (European Coalition for Community Living), etc.

Por su parte, a nivel nacional, también surgen diferentes iniciativas como, por ejemplo, el Consejo Británico de Organizaciones de Personas Discapacitadas o British Council of Organisations of Disabled People (BCODP) en el Reino Unido, el Instituto de Vida Independiente o Independent Living Institute (ILI) en Suecia, el Consejo Alemán de la Discapacidad o Deutschen Behindertenrat (DBR) en Alemania y el Foro de Vida Independiente y Divertad (FVID) en nuestro país.

Los primeros CVI en Europa surgieron en la década de los 80, en países como Reino Unido, Suecia, Alemania, Noruega, Dinamarca, etc., unos ejemplos son ULOBA (Noruega) y STIL (Suecia). En cuanto a las formas jurídicas, las más utilizadas son las asociaciones y las cooperativas sociales (Gómez Jiménez, 2012). Estas organizaciones suelen estar formadas por las propias personas con diversidad funcional, que se organizan para proporcionar los servicios y apoyos que necesitan y también pueden conseguir ventajas para la gestión de servicios a sus asociados, a través de la utilización de las sinergias generadas por la gestión conjunta de los servicios, consiguiendo ahorrar costes (García Alonso, 2003).

\subsection{Movimiento de Vida Independiente en España}

En España, en los años 80, junto a la llegada de la democracia, se produjo un gran desarrollo económico, social, político e institucional, surgiendo las primeras instituciones en defensa de los derechos de las personas con diversidad funcional. No 
fue hasta principios de la década de 2000, cuando desembarcó en territorio español el MVI (Erdtman, 2010). En este sentido, no debemos olvidar que para Tarrow (1997), el cuándo, es decir, el momento del origen de un movimiento social, está íntimamente relacionado con los caracteres que en ese momento posea el sistema y el proceso político, lo que denomina Estructura de Oportunidades Políticas. Dicha estructura empieza a abrirse en 2001, con la creación de la comunidad virtual denominada Foro de Vida Independiente - actualmente Foro de Vida Independiente y Divertad (en adelante FVID)-, cuando se comienza a divulgar y desarrollar la filosofía de este movimiento. El FVID promovió, en 2003, la celebración del I Congreso Europeo sobre Vida Independiente, en Tenerife, y en el que participó como ponente Adolf Ratzka (activista del Movimiento de Vida Independiente en Europa), donde se aprobó el Manifiesto de Tenerife (Erdtman, 2010).

Gracias al activismo del FVID, en los años siguientes se pusieron en marcha distintos proyectos de vida independiente, por ejemplo, el programa de Vida Independiente de Gipuzkoa y las Oficinas de Vida Independiente de Madrid, Barcelona y Galicia. Además, se introdujo en la Ley 39/2006, de 14 de diciembre, de Promoción de la Autonomía Personal y Atención a las Personas en Situación de Dependencia, la figura del/la asistente personal (Urmeneta, 2007).

En España los CVI's reciben el nombre de Oficinas de Vida Independiente (OVI's). En la actualidad, existen cinco en todo el territorio nacional: en Madrid, Barcelona, Galicia, Andalucía y Canarias. A lo largo de esta última década, en nuestro país, se han puesto en marcha algunas iniciativas que ofrecían un servicio de asistencia personal, con el paso del tiempo se ha visto que no han funcionado debido a que no se han basado realmente en los principios y la filosofía del MVI (Gómez Jiménez, 2012).

Para evitar estas situaciones, activistas españoles del MVI han creado una herramienta para evaluar con objetividad las políticas y prácticas desarrolladas bajo la filosofía de este movimiento, denominada Indicador de Vida Independiente (en adelante IVI). Con ella se pretende garantizar que los proyectos de vida independiente sirvan para conseguir verdaderamente una igualdad de oportunidades y para eliminar las discriminaciones que sufren las personas con diversidad funcional debido a sus diferencias.

En el Cuadro 1 se exponen de manera resumida los cambios fundamentales que se persiguen y los indicadores necesarios para medir el nivel de independencia que proporciona un proyecto o iniciativa. 


\section{Cuadro 1}

Cambios fundamentales e indicadores de vida independiente para la evaluación de políticas y acciones desarrolladas bajo la filosofía de vida independiente

\begin{tabular}{|c|c|}
\hline CAMBIOS FUNDAMENTALES & INDICADORES \\
\hline \multirow{5}{*}{$\begin{array}{l}\text { Participación en la vida comu- } \\
\text { nitaria }\end{array}$} & $\begin{array}{l}\text { Las horas de asistencia personal han de estar destinadas a la realización de } \\
\text { todo tipo de actividades }\end{array}$ \\
\hline & $\begin{array}{l}\text { Las horas de asistencia personal han de ser las que necesite la persona be- } \\
\text { neficiaria }\end{array}$ \\
\hline & $\begin{array}{l}\text { Estar abierto a cualquier persona con diversidad funcional, independiente- } \\
\text { mente de su proyecto de vida }\end{array}$ \\
\hline & Se adopten acciones de apoyo para obtener una vivienda accesible \\
\hline & Se adopten acciones de apoyo para conseguir ayudas técnicas \\
\hline \multirow{6}{*}{$\begin{array}{l}\text { Evitar y compensar los déficits } \\
\text { de ciudadanía }\end{array}$} & $\begin{array}{l}\text { Estar abierto a la participación de todas las personas con diversidad funcional, } \\
\text { independientemente de ésta }\end{array}$ \\
\hline & $\begin{array}{l}\text { Estar abierto a la participación de todas las personas con diversidad funcional, } \\
\text { independientemente de su edad }\end{array}$ \\
\hline & Ofrecer formación en filosofía de vida independiente y diversidad \\
\hline & Ofrecer oportunidades y recursos para el apoyo y la consultoría entre iguales \\
\hline & $\begin{array}{l}\text { La libertad que proporciona la asistencia personal no debe ser sufragada eco- } \\
\text { nómicamente por la persona con diversidad funcional }\end{array}$ \\
\hline & $\begin{array}{l}\text { Deben ponderarse las prestaciones económicas para compensar el gasto ex- } \\
\text { traordinario que comporta un entorno discriminatorio }\end{array}$ \\
\hline \multirow{5}{*}{$\begin{array}{l}\text { Centrar las acciones en la per- } \\
\text { sona }\end{array}$} & $\begin{array}{l}\text { La entidad que gestiona y coordina (oficina de vida independiente, coope- } \\
\text { rativa, etc.) ha de estar dirigida por personas con diversidad funcional y con } \\
\text { experiencia propia en vida independiente }\end{array}$ \\
\hline & La persona que coordine los servicios ha de ser usuaria de los mismos \\
\hline & Control de los recursos (pago directo) \\
\hline & Codecisión: Autodeterminación de las necesidades y de los apoyos necesarios \\
\hline & Mecanismo de participación \\
\hline
\end{tabular}

Fuente: Centeno, Lobato y Romañach, 2008, p. 15-24

En definitiva, con el IVI, sus autores han pretendido crear una herramienta de carácter globalizadora que examina el proyecto de vida independiente desde un enfoque 
de derechos que va más allá de un suministro de ayuda económica y de horas de asistencia personal (Centeno, Lobato y Romañach, 2008).

\section{La asistencia personal: recurso humano para la vida independiente}

Aunque Ibarra (2000) nos hace partícipe de lo difícil que es identificar los resultados de un movimiento social con las reivindicaciones del mismo, dada la dificultad metodológica de conocer cuál es la verdadera razón que impulsa a los poderes públicos a adoptar una determinada medida, Ibarra, Martí y Gomá (2002) consideran que los resultados pueden tener que ver con los aspectos más simbólicos o con cambios en los sistemas de valores, (...) o con aspectos más sustantivos, es decir, empujando a cambios de ciertas políticas gubernamentales en marcha, obteniendo derechos sociales o individuales antes no existentes.

De esta manera, podemos observar como el MVI viene a ser un agente de influencia y persuasión que vienen a ensanchar el espacio decisorio en la política pública sobre discapacidad, que es capaz de movilizar y conseguir una reforma o cambio. En este sentido, la asistencia personal (en adelante AP) nace a finales de la década de los 60 del siglo XX, dentro del MVI, como un servicio que proporciona a las personas con diversidad funcional una mayor autonomía e igualdad de oportunidades (Arroyo y Cruz, 2015). Además, desde diciembre de 2006, fecha en la que se aprobó la Convención Internacional sobre los Derechos de las Personas con Discapacidad, la AP es un derecho de todas las personas con diversidad funcional. Viene recogido en su artículo 19 como "Derecho a vivir de forma independiente y a ser incluido en la comunidad” (ONU, 2006).

\subsection{Definición de la asistencia personal}

La AP se considera una herramienta básica para que las personas con diversidad funcional y con necesidades de apoyo, desarrollen en igualdad de condiciones al resto de la población, una vida independiente y de calidad (Rodríguez-Picavea, 2007). Asimismo, es un servicio de apoyo fundamental para que puedan tener un trabajo remunerado, estudiar, participar en actividades de ocio y tiempo libre, etc.

De acuerdo con Nóvoa, Rueda y Hortal (2011), la AP se caracteriza por:

- Ser un servicio de apoyo formal y regularizado mediante un contrato profesional, cuyo fin es que la persona con diversidad funcional y con necesidades de apoyo, pueda realizar las tareas de la vida diaria que por sí sola no puede llevar a cabo. En este sentido, la propia persona con diversidad funcional es la parte contratante y la persona que ejecuta las tareas de asistencia personal, es la parte contratada. 
- Ser una herramienta que favorece su autonomía personal, además de un instrumento básico para su empoderamiento.

- Permitirles asumir la gestión y el control de sus propias vidas.

- Estar reconocido como derecho humano en la Convención Internacional de los Derechos de las Personas con Discapacidad.

Su objetivo fundamental es fomentar la autodeterminación y la vida independiente de todas las personas con diversidad funcional. La AP debe cubrir todas las tareas y actividades que no pueden realizar por sí mismas (Ratzka, 2017).

Las personas destinatarias o beneficiarias de la AP podrán ser cualquiera que tenga necesidad de apoyo para realizar las tareas de la vida diaria, con independencia de su diversidad funcional, edad, situación económica y social. Debe tener un carácter universal, no debe estar condicionado ni restringido a un número de horas determinado, debe tener en cuenta las necesidades, gustos y actividades expresadas por la propia persona.

\subsection{Modalidades de la asistencia personal}

Se puede decir que el/la asistente personal es un/a profesional que ejecuta las tareas que se acuerden en el contrato a cambio de una remuneración económica (Arnau, Rodríguez-Picavea y Romañach, 2007). En el contrato se incluirán todas las actividades diarias como cualquier imprevisto que pueda surgir. Antes del mismo, se deberá realizar un estudio o valoración de las horas y necesidades de asistencia de la persona con diversidad funcional y habrá casos en que estas personas necesitarán más de un/a asistente personal. Todos estos datos deberán quedar plasmados en un documento oficial que, en el caso español, se denomina Plan Individual de Vida Independiente (PIVI) (Consejería de Familia y Asuntos Sociales de la Comunidad de Madrid, 2007).

Para acceder a la AP, existen diferentes fórmulas. Bien, a través de una prestación económica que se le abona directamente a la persona beneficiaria -el denominado pago directo- o mediante un proveedor de servicios que presta la AP (Gómez, Ordax, Moya y Sánchez Santos, 2015).

Mediante una prestación económica o pago directo, la administración pública es la que financia la AP y abona directamente la cuantía económica a la persona con diversidad funcional o a su representante legal. Ésta es la responsable de gestionar su propia asistencia y de rendir cuentas a la administración pública, que le ha concedido dicha prestación económica. En este caso, el asistente personal es contratado directamente por la persona con diversidad funcional y tiene que darle de alta en el correspondiente régimen de la Seguridad Social. Esta es la fórmula más coherente con la filosofía de vida independiente (Gómez, Ordax, Moya y Sánchez Santos, 2015). 
La otra forma de acceder es mediante un proveedor de servicios, que podrá ser una cooperativa o un centro de vida independiente, una empresa privada y, en algunos casos, a través de la propia administración. La administración correspondiente, previo consentimiento de la persona con diversidad funcional o su representante legal, abona la cuantía de la prestación económica a la entidad que ésta haya elegido para prestarle el servicio de asistencia personal. Dicha entidad es la que gestiona la parte administrativa del servicio y está obligada a justificar el pago a la administración correspondiente.

En este sentido, es importante no confundir esta figura laboral con otras. Una diferencia significativa es que las tareas realizadas por el/la asistente personal se adaptan a las verdaderas necesidades y criterios de la persona beneficiaria, cosa que no ocurre en el caso de un/a cuidador/a (Urmeneta, 2011). Extrapolando esta información al caso español, en el Cuadro 2, se presentan las principales diferencias entre la AP y otros tipos de cuidados, como un/a cuidador/a profesional o un/a cuidador/a familiar.

\section{Cuadro 2}

Diferencias entre asistencia personal, cuidador/a profesional y cuidador/a familiar

\begin{tabular}{|l|l|l|}
\hline ASISTENCIA PERSONAL & CUIDADOR PROFESIONAL & CUIDADOR FAMILIAR \\
\hline $\begin{array}{l}\text { La persona beneficiaria es el/ } \\
\text { la "jefe" }\end{array}$ & $\begin{array}{l}\text { El/la “jefe" es una empresa o servicio } \\
\text { ajeno a la persona beneficiaria }\end{array}$ & $\begin{array}{l}\text { Depende de la buena voluntad de } \\
\text { la persona cuidadora }\end{array}$ \\
\hline $\begin{array}{l}\text { Realiza cualquier tarea y/o } \\
\text { función que necesite la perso- } \\
\text { na beneficiaria }\end{array}$ & $\begin{array}{l}\text { Las tareas y/o funciones que realiza se } \\
\text { reducen al ámbito domiciliario. Excep- } \\
\text { cionalmente puede realizar algunas } \\
\text { fuera del hogar como ir a comprar o al } \\
\text { médico }\end{array}$ & $\begin{array}{l}\text { Acuerdo tácito o implícito de qué } \\
\text { cosa se puede pedir y cuál no }\end{array}$ \\
\hline $\begin{array}{l}\text { Se adapta a las necesidades y } \\
\text { directrices de la persona be- } \\
\text { neficiaria }\end{array}$ & $\begin{array}{l}\text { No se adapta a las necesidades y direc- } \\
\text { trices de la persona beneficiaria }\end{array}$ & Dificulta la libre elección \\
\hline \begin{tabular}{l} 
Es un servicio remunerado \\
\hline
\end{tabular} & Es un servicio remunerado & No está remunerado \\
\hline
\end{tabular}

Fuente: Elaboración propia a partir de Xavier Urmeneta, 2011

El MVI considera que la AP es un servicio de apoyo al empoderamiento de todas las personas con diversidad funcional, ajustándose a sus verdaderas necesidades, ya sean para las actividades de la vida diaria, laborales, educativas, de participación social, de ocio y tiempo libre (ENIL, 1989). 


\section{Consideraciones finales}

Con el concepto personas con diversidad funcional se engloba a un grupo de personas con algunas características físicas, psíquicas y/o sensoriales como consecuencia de una malformación, enfermedad o accidente que conforman un grupo muy heterogéneo. Sin embargo, todas tienen en común su experiencia con la discriminación y la opresión, ya que ven limitados su desarrollo personal, su autonomía y su inclusión en la sociedad.

Con el nacimiento y desarrollo del MVI, las personas con diversidad funcional tomaron conciencia de sus derechos. Uno de éstos, es el derecho a vivir de forma independiente y en igualdad de oportunidades al resto de la ciudadanía y para hacerlo realidad, la AP es imprescindible.

En la década de los 90, con la llegada del MVI a Europa, gracias a sus demandas, se pusieron en marcha diferentes proyectos de AP y se aprobaron las primeras normativas, como la Convención Internacional sobre los Derechos de las Personas con Discapacidad de las Naciones Unidas que en su artículo 19, reconoce la AP como un derecho (ONU, 2006).

Este recurso tiene ya más de 50 años de historia y casi 40 de establecimiento en Europa, sin embargo, en nuestro país la implantación de la AP ha sido tardía, no fue hasta el año 2006.

Es cierto que, en los últimos años, en España, se ha producido un auge en el interés por el tema de la AP que se evidencia en el número de proyectos puestos en marcha. En general, aún es un recurso poco conocido. En este sentido y debido a esta carencia, el presente estudio es fundamental para conocer la realidad de un movimiento social y un recurso humano que favorecen la autodeterminación, participación y calidad de vida de las personas con diversidad funcional.

\section{Bibliografía}

ARNAU RIPOLLÉS, María Soledad, RODRÍGUEZ-PICAVEA, Alejandro y ROMAÑACH CABRERO, Javier. (2007). "Asistencia personal para la vida independiente y la promoción de la autonomía de las personas con diversidad funcional (discapacidad) en España". Foro de Vida Independiente y Divertad. Disponible en https://uplalaunion.org/wp-content/uploads/2020/04/Asistente_ personal.pdf [Consultado 25-11-2019].

ARNAU RIPOLLÉS, María Soledad. (2006). "Ley de la "In-Dependencia": Pasos necesarios para garantizar un nuevo Derecho de Ciudadanía". Lan harremanak: Revista de relaciones laborales, (15), 41-64. Disponible en https://dialnet.unirioja. es/servlet/articulo? codigo=2378144 [Consultado 15-11-2019]. 
ARNAU RIPOLLÉS, María Soledad. (2013). “La Filosofía de Vida Independiente. Una Estrategia Política Noviolenta para una Cultura de Paz". Revista Latinoamericana de Educación Inclusiva, 7(1), 93-112. Disponible en https://dialnet.unirioja.es/ servlet/articulo? codigo $=4268728$ [Consultado 5-10-2019].

ARROYO MENDEZ, Javier y CRUZ GONZÁLEZ, Elena. (2015). "La asistencia personal", en E. ORTEGA ALONSO (coord.), Situación de la Asistencia Personal en España, Madrid, PREDIF, pp. 12-17.

CAMPO BLANCO, Maribel. (2005). "Orígenes e historia del Movimiento de Vida Independiente. Vida Independiente en la Universidad", en I Jornada de Vida Independiente, Donostia, Diputación Foral de Gipuzkoa.

CENTENO, Antonio, LOBATO Manuel y ROMAÑACH, Javier. (2008). “Indicadores de Vida Independiente (IVI) para la evaluación de políticas y acciones desarrolladas bajo la filosofía de Vida Independiente". Foro de Vida Independiente y Divertad. Disponible en https://studylib.es/doc/7216923/indicadores-de-vidaindependiente--ivi--para-la-evaluaci\%C3\%B3... [Consultado 5-1-2020].

CONSEJERÍA DE FAMILIA Y ASUNTOS SOCIALES DE LA COMUNIDAD DE MADRID. (2007). Guía Práctica de la Asistencia Personal. Disponible en: http:// www.madrid.org/bvirtual/BVCM013822.pdf [Consultado 27-10-2019].

DALTON, Russell, KUECHLER, Manfred y BÜRKLIN, Wilhelm. (1992). "El reto de los nuevos movimientos", en R. DALTON y M. KUECHLER. (eds.), Los nuevos movimientos sociales: un reto al orden internacional, Valencia, Alfons el Magnànim, pp. 19-42.

DEJONG, Gerben. (1979). The Movement for independent living: Origins, ideology and implications for disability research. University Centers for International Rehabilitation, East Lansing, Michigan, Michigan State University.

ERDTMAN, E. (2010). "Spain-Assistance-available in Spain by law, but not in reality". Independent Living Institute (ILI). Disponible en http://www.independentliving. org/docs7/Spain-personal-assistance-not-reality.html [Consultado 24-10-2019].

EUROPEAN NETWORK ON INDEPENDENT LIVING (ENIL) (1989). "The Strasbourg Resolutions". Independent Living Institute (ILI). Disponible en https:// www.independentliving.org/docs2/enilstrasbourgresolutions.html [Consultado 8-10-2019].

GARCÍA ALONSO, J. Vidal. (coord.). (2003). El Movimiento de Vida Independiente. Experiencias Internacionales. Madrid, Fundación Luis Vives.

GÓMEZ JIMÉNEZ, Nuria y ARROYO, Javier. (2013). "Oficinas de Vida Independiente”. Revista Latinoamericana de Educación Inclusiva, 7(1), 79-92.

GÓMEZJIMÉNEZ, Nuria. (2012). "Vida Independiente: Instrumentos para garantizar el cambio de paradigma”, en A. PIÉ BALAGUER. (coord.), Deconstruyendo la 
dependencia: Propuesta para una vida independiente, Barcelona, Editorial UOC, pp. $185-212$.

GÓMEZ, Nuria, ORDAX, Elena, MOYA, Ma José y SÁNCHEZ SANTOS, Fernando. (2015). “Prestación del servicio de asistencia personal", en E. ORTEGA ALONSO (coord.), Situación de la Asistencia Personal en España, Madrid, PREDIF, pp. 26-34.

HASLER, Frances. (2003). "Vida Independiente: Visión filosófica”, en J. V. GARCÍA ALONSO (coord.), El movimiento de vida independiente. Experiencias internacionales, Madrid, Fundación Luis Vives, pp. 55-61.

IÁÑEZ DOMÍNGUEZ, Antonio. (2009). "Vida Independiente y Diversidad Funcional. Resultados de una Investigación Social Aplicada en la Provincia de Sevilla”. Portularia IX(1), 93-103.

IÁÑEZ DOMÍNGUEZ, Antonio. (2010). Prisioneros del cuerpo. La construcción social de la diversidad funcional. A Coruña, Diversitas Ediciones.

IBARRA, Pedro, MARTI, Salvador y GOMÁ, Ricard. (2002): Creadores de democracia radical: Movimientos sociales y redes de políticas públicas. Barcelona, Icaria.

IBARRA, Pedro. (2000). “¿Qué son los movimientos sociales?”, en E. GRAU y P. IBARRA (eds.), Anuario de movimientos sociales. Participando en la Red, Barcelona, Icaria, pp. 1-9.

Ley 39/2006 de Promoción de la Autonomía Personal y Atención a las Personas en Situación de Dependencia. Boletín Oficial del Estado (BOE), núm. 299, de 15 de diciembre de 2006, pp. 44142-44156.

LOBATO GALINDO, Manuel. (2005). "Filosofía del Movimiento de Vida Independiente. El concepto de Asistencia Personal", en I Jornada de Vida Independiente, Donostia, Diputación Foral de Gipuzkoa.

MADRID PÉREZ, Antonio. (2013). "Nothing About Us Without Us! El Movimiento de Vida Independiente: Comprensión, Acción y Transformación Democrática”, Oxímora. Revista Internacional de Ética y Política, (2), pp. 22-38. Disponible en: https://revistes.ub.edu/index.php/oximora/article/view/6292/8034 [Consultado 29-9-2019].

MARAÑA GONZÁLEZ, Juan José y RATZKA, Adolf. (1999). Vida Independiente. Buenas Prácticas. Madrid, Imserso.

MARAÑA GONZÁLEZ, Juan José. (2004). Vida independiente. Nuevos modelos organizativos. Santiago de Compostela, A Coruña, Asociación Iniciativas y Estudios Sociales. Disponible en: http://forovidaindependiente.org/wp-content/ uploads/vida_independiente_nuevos_modelos.pdf [Consultado 12-12-2019].

NÓVOA ROMAY, Juan Antonio, RUEDA BRENES, Miguel y HORTAL JAPÓN, Coral. (2011). "La asistencia personal. Un recurso económicamente eficiente, 
socialmente y humanamente deseable, y excelente en el empleo". Foro de Vida Independiente y Divertad. Disponible en: http://forovidaindependiente.org/ wp-content/uploads/residencias_asistente.pdf [Consultado 23-11-2019].

NÓVOA ROMAY, Juan Antonio. (2003). "Aproximación al modelo de Centro de Vida Independiente en los Estados Unidos de Norteamérica". Foro de Vida Independiente y Divertad. Disponible en: http://forovidaindependiente.org/ aproximacion-al-modelo-de-centro-de-vida-independiente-en-los-estadosunidos-de-norteamerica/ [Consultado 18-10-2019].

ORGANIZACIÓN DE LAS NACIONES UNIDAS (ONU). (2006). Convención Internacional sobre los derechos de las personas con Discapacidad. Disponible en: https://www.un.org/disabilities/documents/convention/convoptprot-s.pdf [Consultado 10-1-2020].

PALACIOS, Agustina y ROMAÑACH, Javier. (2006). El modelo de la diversidad. La Bioética y los Derechos Humanos como herramientas para alcanzar la plena dignidad en la diversidad funcional. Santiago de Compostela, Ediciones Diversitas-AIES. Disponible en: https://sid.usal.es/idocs/F8/FDO18989/ modelo_diversidad.pdf [Consultado 4-12-2019].

RATZKA, Adolf. (2017). "Self-determination for Persons with Extensive Disabilities through Direct Payments for Personal Assistance". Independent Living Institute (ILI). Disponible en: https://www.independentliving.org/docs7/Selfdetermination-direct-payments.html [Consultado 19-11-2019].

RODRÍGUEZ-PICAVEA MATILLA, Alejandro. (2007). "Reflexiones en torno a la figura del asistente personal para la vida independiente y la promoción de la autonomía de las personas con diversidad funcional (discapacidad)". Zerbitzuan Revista de Servicios Sociales, (41), 115-127. Disponible en: http://riberdis.cedd. net/bitstream/handle/11181/2814/reflexiones_figura_asistente_personal_para_ la_vida_independiente.pdf? sequence $=1 \& \mathrm{rd}=0031750605219128 \quad$ [Consultado 30-10-2019].

RODRÍGUEZ-PICAVEA MATILLA, Alejandro. (2010). "Vida Independiente: Dignidad y derechos de la Diversidad Funcional". TOG [Revista en Internet], 7(6), 78-101. Disponible en: http://www.revistatog.com/suple/num6/picavea. pdf [Consultado 25-11-2019].

TARROW, Sidey. (1997). El poder en movimiento: los movimientos sociales, la acción colectiva y la política. Madrid, Ed. Alianza.

URMENETA SANROMÁ, Xabier. (2007). "La vida independiente en Gipuzkoa: Una alternativa para las personas con discapacidad y dependencia”. Zerbitzuan Revista de Servicios Sociales, (42), 87-92. 
URMENETA SANROMÁ, Xabier. (2011). "Vida independiente y asistencia personal. La experiencia de Guipuzkoa", en Jornada Asistencia Personal y Promoción de la Autonomía, Oviedo y Bilbao, Red Vía Oviedo y European Network on Independent Living (ENIL) ${ }^{3}$. 
